\title{
Conditioning the eyeblink response to sound in infants '
}

\author{
LARRY M. LINTZ, HIRAM E. FITZGERALD AND YVONNE BRACKBILL
}

UNIVERSTTY OF DENVEF

Eyeblink conditioning to a complex sound was investigated in eight experimental and 12 control infants, with a median age of 69.5 days. All eight experimental Ss reached criterion of nine blinks on 10 successive trials. In control groups for spontaneous blinking, for effects of presentations of CS alone, and for effects of unpaired presentations of CS and UCS, no $S$ approached the criterion of conditioning.

In the United States, the eyeblink as a CR has been used extensively in research with adult human beings but has rarely been used with younger Ss and never successfully with infants. Nevertheless, the eyeblink has considerable potential usefulness for research on defensive conditioning in infancy because it is such a mild representative of that class of responses. In addition, infants are potentially useful Ss for eyeblink conditioning research because the cognitive-attitudinal factors that plague the study of eyeblink conditioning in adults have not yet appeared. The purpose of the present study was to investigate the course of eyeblink conditioning in young infants.

Ss were 20 healthy, full term infants ranging in age at the beginning of the experiment from 33 days to 133 days (median, 69.5). There were eight Ss in the experimental group and four Ss in each of the three control groups. Sessions were carried out only when infants were in a state of quiet alertness. ${ }^{2}$ The UCS was an air puff of 0.3 sec. duration delivered at 2 psi pressure from a tube the end of which was 2.5 in. distant from S's right eye. The CS was a tape-recorded $65 \mathrm{~dB}$ complex sound with a duration of $0.20 \mathrm{sec}$.

The procedure for the experimental group was as follows: The CS was presented at random intervals ranging from 30 to $60 \mathrm{sec}$. (mean, 39.2). The interstimulus interval was 1.00 sec. Test trial presentations of the CS alone were randomly interspersed among the conditioning trials in a $1: 3$ ratio representing, in effect, a partial reinforcement procedure. This was used instead of the regular reinforcement procedure generally employed in adult eyeblink conditioning first, because of the lack of normative data on infant eyeblink latencies that must be known in order to record anticipatory blinking on reinforced trials, and second, because Koltsova (1967) has reported "extinction during reinforcement" when regular reinforcement is used in conditioning blinking in young children.

In adult conditioning the polygraph record is commonly used as the single referent of blinking, but in infant work we have found it advisable to record eyeblinks both by an observer (O) and by an instrumented magnetic detector which uses a magnet fastened to S's upper eyelid (Lintz \& Fitzgerald, 1966). An eyeblink was defined as a full closure of the eyelid rather than the partial excursion generally used in adult studies. In deciding whether or not a blink occurred, blind scoring across 200 trials from four different $\mathrm{Ss}$ indicated $97 \%$ agreement between these two methods. Of the six cases of disagreement, five were scored as blinks from the magnet record but not by $O$. Thus $O$ gave slightly more conservative judgments, and in the final scoring $O$ 's record was used to determine occurrence of eyeblinks. Time of occurrence of blinks was determined from the magnet record. On any test trial, $S$ was credited with a $C R$ if $O$ had indicated an eyeblink and if the magnet record indicated that that blink began during the $1.3 \mathrm{sec}$. following CS onset.

Daily conditioning sessions, carried out in a soundproof booth, consisted of not more than 25 trialsthis having been found in pilot work to be about the limit of tolerant cooperation for infant Ss. Conditioning continued to a criterion of nine CRs out of 10 successive test trials. This represents a more stringent criterion than is usually employed in adult conditioning studies, and whether the feasibility of its use in this case reflects a real difference in ease of conditioning or limits of conditionability is not clear at present.

Procedure for the three control groups differed from that used for the experimental group as follows. In Control I, the control group of major interest, four Ss received the same total number of CS and UCS presentations-though unpaired in this instance-as the four slowest conditioners of the experimental group had received. This took from 15 to 18 daily sessions (median, 16.5). Controls II and III were carried out in single sessions that lasted as long as $S$ could be maintained in a state of quiet alertness. Median session length was $23 \mathrm{~min}$. for Control II and $26 \mathrm{~min}$. for Control III. The four Ss in Control II received random-interval presentations of CS only. The four Ss in Control III were used to obtain a measure of spontaneous blinking; they received no CS or UCS presentations. For Ss in this group, a "test trial" blink was scored for any lid closure occurring during the 1.3-sec. intervals that corresponded in time with the test trials for Ss in Control II. 


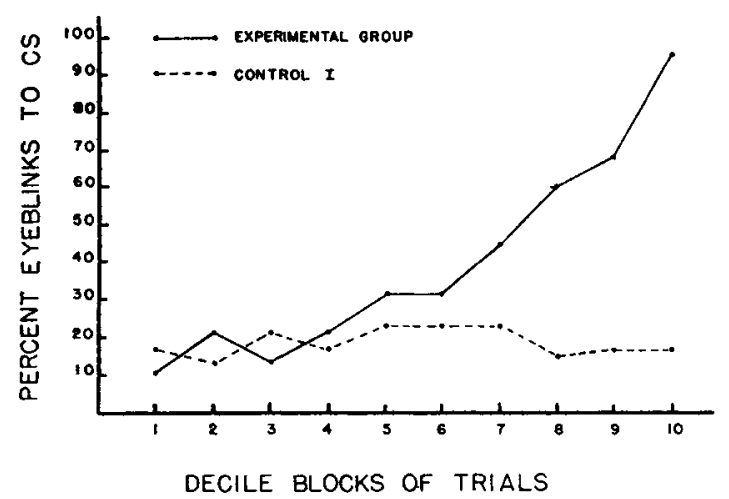

Fig. 1. Test trial results for the experimental group and major control group.

Test trial results for the experimental group and Control I, as shown in Fig. 1, were plotted in "decile" blocks, each such block consisting of one-tenth the total test trials for each $S$. The frequency of blinking is relatively constant over trials for Control I whereas it increases markedly for the experimental group. For the last test trial block a test of the difference between groups yielded a $\mathrm{t}$ of 8.06 ( $\mathrm{df}=10, \mathrm{p}<.001$ ). Evidently eyeblinking can be successfully conditioned to a sound CS in infants as young as those used in the present study.

There is also an apparent difference among the three control groups. The probability of a blink on any test trial was .362 for Control I, .084 for Control II, and .058 for Control III. Thus it appears that administration of the UCS is the most important pseudoconditioning control component for this conditioning situation. In addition, it should be noted that the higher probability figure for blinking in Control II Ss as compared to Control III Ss was due to a preponderance of blinks on the first or second stimulus presentation-a typical manifestation of the orienting reflex and one that extinguished in all cases by the fourth application of the CS.

\section{References}

Koltsova, M. M. The physiological mechanisms underlying the development of generalization in the child. In Y. Brackbill and G. G. Thompson (Eds.), Behavior in infancy and early childhood: $A$ book of readings. New York: Free Press, 1967.

Lintz, L. M., \& Fitzgerald, H. E. Apparatus for eyeblink conditioning in infants. J. exp. child Psychol., 1966, 4, 276-279.

\section{Notes}

1. This research was supported in part by NSF research grant GB4784. It was also supported in part by an NSF predoctoral research fellowship, a USPHS Traineeship MH-8297, and a research career award 5-K3-MH-5925 to the first, second, and third authors, respectively.

2. Stage 4 on a 5-point scale of state ranging from quiet sleep (1) to agitated-awake (5). Independent, time-sample observations of infants between 7 and 56 days of age by 3 observers showed $98 \%$ agreement on use of this scale. 